\title{
Latest inflation model constraints from cosmic microwave background measurements: Addendum
}

\author{
William H. Kinney* \\ Department of Physics, University at Buffalo, the State University of New York, Buffalo, New York 14260-1500, USA
}

Edward W. Kolb ${ }^{+}$

Department of Astronomy and Astrophysics, Enrico Fermi Institute, and Kavli Institute for Cosmological Physics, University of Chicago, Chicago, Illinois 60637-1433

Alessandro Melchiorri ${ }^{\ddagger}$

Dipartimento di Fisica and Sezione INFN, Universita' di Roma “La Sapienza”, Ple Aldo Moro 2, 00185, Italy

Antonio Riotto $\$$

CERN, Theory Division, Geneva 23, CH-1211, Switzerland and INFN, Sezione di Padova, Via Marzolo 8, I-35131, Padova, Italy

(Received 1 September 2008; published 9 October 2008)

\begin{abstract}
In this addendum to Phys. Rev. D 74, 023502 (2006), we present an update of cosmological constraints on single-field inflation in light of the Wilkinson Microwave Ansiotropy Probe satellite mission five-year results (WMAP5). We find that the cosmic microwave background data are quite consistent with a Harrison-Zel'dovich primordial spectrum with no running and zero tensor amplitude. We find that the three main conclusions of our analysis of the WMAP three-year data (WMAP3) are consistent with the WMAP5 data: (1) the Harrison-Zel'dovich model is within the 95\% confidence level contours; (2) there is no evidence for running of the spectral index of scalar perturbations; (3) from the WMAP 5 data alone, potentials of the form $V \propto \phi^{p}$ are consistent with the data for $p=2$ and are ruled out for $p=4$. Furthermore, consistent with our WMAP3 analysis, we find no evidence for primordial tensor perturbations, this time with a 95\% confidence upper limit of $r<0.4$ for the WMAP5 data alone, and $r<0.35$ for the WMAP5 data taken in combination with the Arcminute Cosmology Bolometer Array (ACBAR).
\end{abstract}

DOI: 10.1103/PhysRevD.78.087302

PACS numbers: $98.80 . \mathrm{Cq}$

\section{INTRODUCTION}

In this addendum to [1] we update the constraints on various single-field inflationary models in the light of recent cosmological data. We refer the reader to Refs. [1,2] for a more detailed discussion of the inflationary model space, and for more extensive references. In particular, we compare our results to those reported by the WMAP team in Komatsu et al., [3] and in Dunkley et al. [4]. As first pointed out in Ref. [5], for single-field inflation models, the relevant parameter space for distinguishing among models is defined by the scalar spectral index $n$, the ratio of tensor-to-scalar fluctuations $r$, and the running of the scalar spectral index $d n / d \ln k$. The Harrison-Zeldovich (HZ) point $(n=1, r=0)$ is comfortably within the $95 \%$ C.L. contour of Ref. [3], and just at the borderline of the $95 \%$ C.L. contour of Ref. [4]. This small difference appears to be statistically insignificant. In our analysis using the WMAP5 data with slightly different priors than used by the WMAP team (discussed in

\footnotetext{
*whkinney@buffalo.edu

${ }^{+}$Rocky.Kolb@uchicago.edu

¥alessandro.melchiorri@ roma1.infn.it

§antonio.riotto@pd.infn.it
}

Sec. III) we find that the HZ model is not preferred, but neither is it strongly disfavored.

The paper is organized as follows. In Sec. II we quickly review single-field inflation models and their observables and define the inflationary model space as a function of the slow-roll parameters $\epsilon$ and $\eta$. In Sec. III we describe our analysis method as well as our results. Since a study of the implications of the WMAP5 data for single-field models of inflation has been already performed by the WMAP Collaboration themselves [3], we will also specify the differences between our analysis and theirs. In Sec. IV we present our conclusions.

\section{SINGLE-FIELD INFLATION AND THE INFLATIONARY OBSERVABLES}

Inflation not only explains the large-scale homogeneity of the Universe, but also provides a mechanism for explaining the observed level of inhomogeneity as well. During inflation, quantum fluctuations on small scales are quickly redshifted to scales much larger than the horizon size, where they are "frozen" as perturbations in the background metric. The metric perturbations created during inflation are of two types: scalar, or curvature perturbations, which couple to the stress-energy of matter in the 
Universe and form the "seeds" for structure formation, and tensor, or gravitational-wave perturbations, which do not couple to matter. Both scalar and tensor perturbations contribute to $\mathrm{CMB}$ anisotropies. Scalar fluctuations can also be interpreted as fluctuations in the density of the matter in the Universe. Scalar fluctuations can be quantitatively characterized by the comoving curvature perturbation $P_{\mathcal{R}}$. As long as slow roll is attained, the curvature (scalar) perturbation at horizon crossing can be shown to be [6]

$$
P_{\mathcal{R}}^{1 / 2}(k)=\left(\frac{H^{2}}{2 \pi \dot{\phi}}\right)_{k=a H}=\left[\frac{H}{m_{\mathrm{Pl}}} \frac{1}{\sqrt{\pi \epsilon}}\right]_{k=a H} .
$$

Here, $\phi$ denotes the slow-rolling scalar field dominating the energy density of the Universe during inflation, the socalled inflaton, $H$ is the Hubble rate, and $m_{\mathrm{Pl}}=$ $1.2 \times 10^{19} \mathrm{GeV}$ is the Planck scale. The slow-roll approximation is consistent if both the slope and curvature of the inflaton potential $V(\phi)$ are small (in Planckian units), $V^{\prime}$, $V^{\prime \prime} \ll V$. In this case the slow-roll parameter $\epsilon$ can be expressed in terms of the potential as

$$
\epsilon \equiv \frac{m_{\mathrm{Pl}}^{2}}{4 \pi}\left(\frac{H^{\prime}(\phi)}{H(\phi)}\right)^{2} \simeq \frac{m_{\mathrm{Pl}}^{2}}{16 \pi}\left(\frac{V^{\prime}(\phi)}{V(\phi)}\right)^{2} .
$$

We will also need in the following a second "slow-roll parameter" $\eta$ defined by

$$
\eta(\phi) \equiv \frac{m_{\mathrm{Pl}}^{2}}{4 \pi}\left(\frac{H^{\prime \prime}(\phi)}{H(\phi)}\right) \simeq \frac{m_{\mathrm{Pl}}^{2}}{8 \pi}\left[\frac{V^{\prime \prime}(\phi)}{V(\phi)}-\frac{1}{2}\left(\frac{V^{\prime}(\phi)}{V(\phi)}\right)^{2}\right] .
$$

Slow roll is then a consistent approximation for $\epsilon, \eta \ll 1$.

The fluctuation power spectrum is, in general, a function of wave number $k$, and is evaluated when a given mode crosses outside the horizon during inflation, $k=a H$. Outside the horizon, modes do not evolve, so the amplitude of the mode when it crosses back inside the horizon during a later radiation- or matter-dominated epoch is just its value when it left the horizon during inflation. Instead of specifying the fluctuation amplitude directly as a function of $k$, it is convenient to specify it as a function of the number of $e$-folds $N$ before the end of inflation at which a mode crossed outside the horizon.

The scalar spectral index $n$ for $P_{\mathcal{R}}$ is defined by

$$
n-1 \equiv \frac{d \ln P_{\mathcal{R}}}{d \ln k}
$$

so that a scale-invariant spectrum, in which modes have constant amplitude at horizon crossing, is characterized by $n=1$.

The power spectrum of tensor fluctuation modes and the corresponding tensor spectral index is given by [6]

$$
P_{T}^{1 / 2}\left(k_{N}\right)=\left[\frac{4 H}{m_{\mathrm{Pl}} \sqrt{\pi}}\right]_{k=a H}, \quad n_{T} \equiv \frac{d \ln P_{T}}{d \ln k} .
$$

The ratio of tensor-to-scalar modes is then $P_{T} / P_{\mathcal{R}}=$ $16 \epsilon$, so that tensor modes are negligible for $\epsilon \ll 1$. In the limit of slow roll, the spectral indices $n$ and $n_{T}$ vary slowly or not at all with scale. We can write the spectral indices $n$ and $n_{T}$ to lowest order in terms of the slow-roll parameters $\epsilon$ and $\eta$ as

$$
n \simeq 1-4 \epsilon+2 \eta, \quad n_{T} \simeq-2 \epsilon .
$$

The tensor/scalar ratio is frequently expressed as a quantity $r$, which is conventionally normalized as

$$
r \equiv 16 \epsilon=\frac{P_{T}}{P_{\mathcal{R}}} .
$$

The tensor spectral index is not an independent parameter, but is proportional to the tensor/scalar ratio, given to lowest order in slow roll by $n_{T} \simeq-2 \epsilon=-r / 8$. This is known as the consistency relation for inflation. A given inflation model can therefore be described to lowest order in slow roll by three independent parameters: $P_{\mathcal{R}}, P_{T}$, and $n$. If we wish to include higher-order effects, we would have to include a fourth parameter describing the running of the scalar spectral index, $d n / d \ln k$.

\section{ANALYSIS AND RESULTS}

The method we adopt is based on the publicly available Markov chain Monte Carlo (MCMC) package COSMOMC [7]. We sample the same eight-dimensional set of cosmological parameters and we adopt the same analysis method of [1]. However, we now analyze the five-year data [3] (temperature and polarization) with the routine for computing the likelihood supplied by the WMAP team [8]. We also perform an analysis based on the WMAP five-year data in combination with results from the Arcminute Cosmology Bolometer Array (ACBAR) [9], considered up to multipole $\ell=2000$. The constraints are obtained by using the standard COSMOMC procedure based on sample counts, which allow for non-Gaussianity of the posterior distribution.

We perform the analysis in two ways: first, we constrain parameters with a prior assumption of $d n / d \ln k=0$, i.e., an exact power-law perturbation spectrum, and second, with $d n / d \ln k$, a free parameter in the MCMC.

All fits are performed at a pivot scale of $k=0.017$, which is different from our previous analysis and the analysis of Dunkley et al. and Komatsu et al., all of which are normalized to a pivot scale of $k=0.002$. The reason for the different choice of pivot scale is to mitigate the parameter degeneracy between $n$ and $d n / d \ln k$ [10], which significantly improves convergence of the MCMC code. In the case of $d n / d \ln k=0$, the choice of pivot scale has no effect on the spectral index $n$, since a change of pivot affects only the normalization:

$P(k)=P_{0.002}\left(\frac{k}{k=0.002}\right)^{n-1}=P_{0.017}\left(\frac{k}{k=0.017}\right)^{n-1}$.

However, the change of pivot results in a rescaling of the tensor/scalar ratio $r$ which is dependent on $r$ and $n$, since 


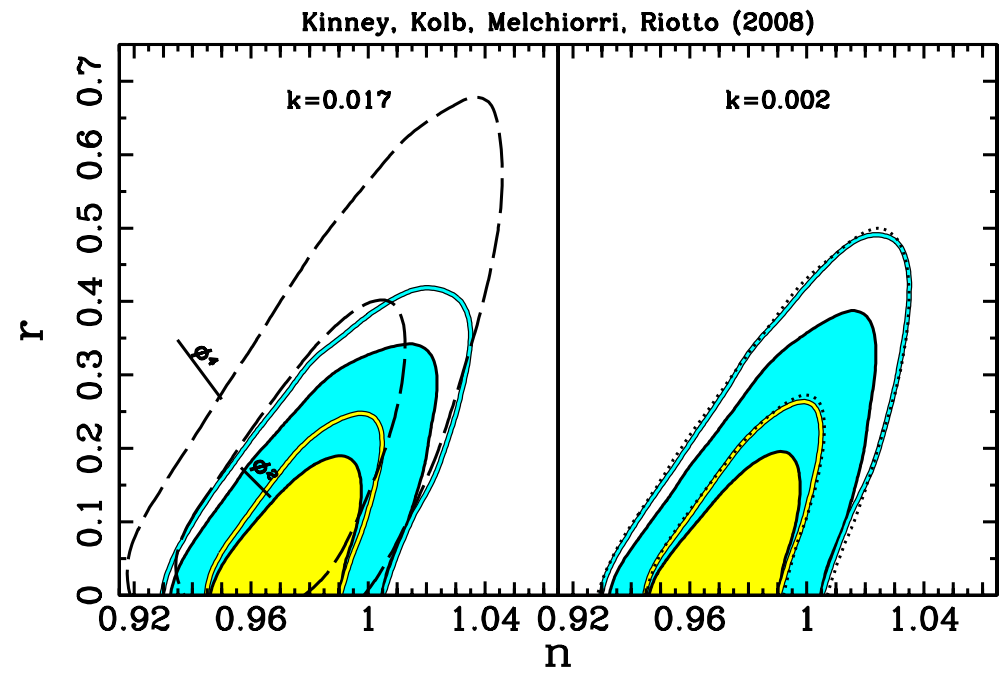

FIG. 1 (color online). 68\% C.L. and 95\% C.L. contours in the $n, r$ parameter space for WMAP5 alone (open contours) and WMAP5 + ACBAR (filled contours), with a prior of $d n / d \ln k=0$. In the left figure the line segments show the predictions for $V(\phi)=m^{2} \phi^{2}$ (lower line segment) and $V(\phi)=$ $\lambda \phi^{4}$ (upper line segment) for the number $N$ of $e$-folds before the end of inflation at which a mode crossed outside the horizon in the range $N=[46,60]$. Also in the left figure the dashed lines show the $68 \%$ C.L. and $95 \%$ C.L. contours from our previous analysis of the WMAP 3-year data. The figure on the left is for a pivot scale of $k=0.017$ and the figure on the right is the same contours translated to a pivot scale of $k=0.002$ by Eq. (10). The dotted line in the right panel is the result of a MCMC analysis run directly at a pivot of $k=0.002$ using the WMAP5 data alone.

$$
r(k)=\frac{P_{T}(k)}{P_{\mathcal{R}}(k)} \propto k^{n_{T}+(1-n)},
$$

and therefore, using the consistency relation $n_{T}=-r / 8$,

$$
r_{0.017}=\left.\frac{P_{T}}{P_{\mathcal{R}}}\right|_{k=0.017}=r_{0.002}\left(\frac{0.002}{0.017}\right)^{n-1+r_{0.002} / 8}
$$

Figure 1 shows $68 \%$ and $95 \%$ confidence limits on $r$ and $n$ in the case of a no-running prior, $d n / d \ln k=0$. Clearly the choice of the pivot scale makes very little difference in this case (and since it is just a normalization for $r$, no difference at all in the $r=0$ case).

In Fig. 2 we show the $68 \%$ and $95 \%$ confidence limits on $r$ and $n$ in the case where we allow running of the spectrum $d n / d \ln k$ as a free parameter. Again the WMAP5 alone

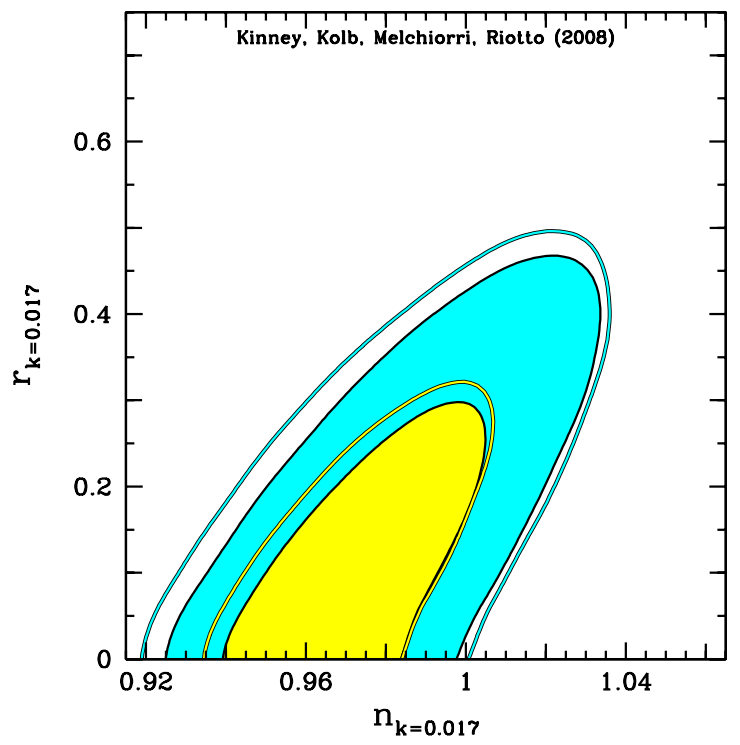

FIG. 2 (color online). $68 \%$ and $95 \%$ confidence limits on $r$ and $n$ for WMAP5 alone (open contours) and WMAP5 + ACBAR (filled contours) allowing the possibility of a running spectral index.
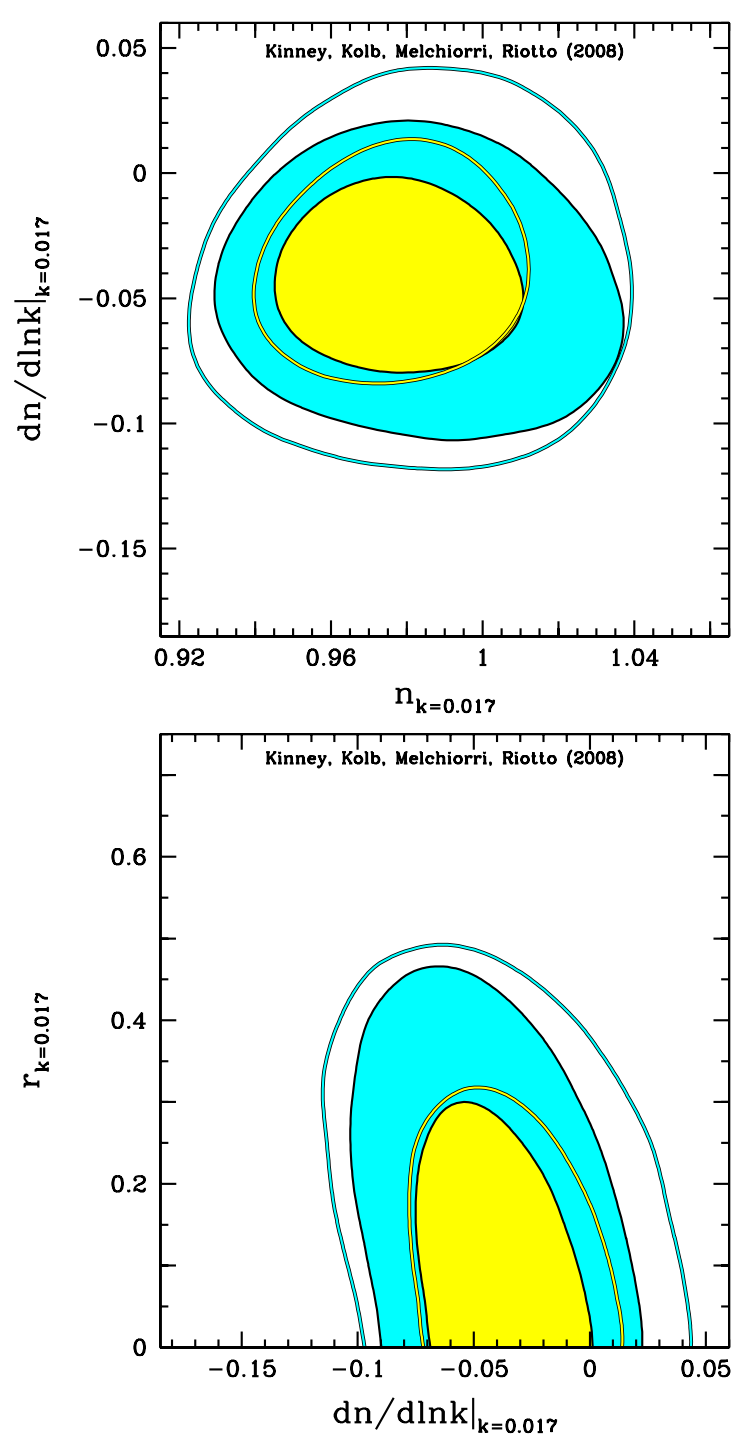

FIG. 3 (color online). $68 \%$ and 95\% confidence limits for $d n / d \ln k$ vs $n$ and $r$ vs $d n / d \ln k$. The different contours are for different data sets as in Figs. 1 and 2. 
data correspond to the open contours and WMAP5 + ACBAR to the filled contours.

Finally in Fig. 3 we show the $68 \%$ and $95 \%$ confidence limits on $d n / d \ln k$ as a function of $n$ and $r$ as a function of $d n / d \ln k$.

\section{CONCLUSIONS}

In this paper we presented an analysis of the recent WMAP five-year data set with an emphasis on parameters relevant for distinguishing among the various possible models for inflation. This analysis is an update to our similar analysis of the WMAP three-year data set [1]. Here we reach three main conclusions, which are either unchanged or slightly strengthened with respect to [1]: (1) As we can see from Fig. 1, the Harrison-Zel'dovich model is not preferred by the data but is within the $95 \%$ confidence level contour. In particular we found that, considering the whole sets of models in the "no-running" 7-D chain, the $\mathrm{HZ}$ best fit model is at $\Delta \chi^{2} / 2=3.3$ with respect to the overall best fit. When running is included, the $\mathrm{HZ}$ best fit model is at $\Delta \chi^{2} / 2=3.9$ with respect to the overall best fit. Since $\Delta \chi^{2} / 2=6.4$ at $95.4 \%$ confidence level for 6 degrees of freedom, those numbers clearly indicate that the HZ spectrum is in reasonable agreement with the WMAP five-year data. (2) There is no evidence for running of the spectral index of scalar perturbations. Figure 3 shows likelihood contours for $d n / d \ln k$ relative to the parameters $n$ and $r$, calculated at a pivot scale of $k=$
0.017 to minimize parameter degeneracies, showing that the case of a pure power-law spectrum $d n / d \ln k=0$ is fully consistent with all data sets. (3) Potentials of the form $V \propto \phi^{p}$ are consistent with the data for $p=2$, and are ruled out by the WMAP five-year data alone for $p=4$. This is a stronger conclusion that was possible with the WMAP three-year data, which was marginally consistent with $\lambda \phi^{4}$ at a $95 \%$ C.L. We find no evidence for a nonzero tensor/scalar ratio, with a $95 \%$ C.L. upper limit of $r<0.4$ for the WMAP five-year data alone, and $r<0.35$ for WMAP in combination with ACBAR. This contradicts claims of a lower bound on $r$ in Ref. [11]. Our results are consistent with the results of Peiris and Easther in Ref. [12].

\section{ACKNOWLEDGMENTS}

We acknowledge support provided by the Center for Computational Research at the University at Buffalo. W.H.K. is supported in part by the National Science Foundation under Grant No. NSF-PHY-0456777. This research was supported in part by the Department of Energy and the European Community's Research Training Networks under Contracts No. MRTN-CT-2004-503369 and No. MRTN-CT-2006-035505. This research has been supported by ASI Contract No. I/016/07/0 "COFIS." We thank Joanna Dunkley and Eichiro Komatsu for helpful conversations.
[1] W. H. Kinney, E. W. Kolb, A. Melchiorri, and A. Riotto, Phys. Rev. D 74, 023502 (2006).

[2] W. H. Kinney, E. W. Kolb, A. Melchiorri, and A. Riotto, Phys. Rev. D 69, 103516 (2004).

[3] E. Komatsu et al., arXiv:0803.0547.

[4] J. Dunkley et al., arXiv:0803.0586.

[5] S. Dodelson, W. H. Kinney, and E. W. Kolb, Phys. Rev. D 56, 3207 (1997).

[6] D. H. Lyth and A. Riotto, Phys. Rep. 314, 1 (1999); W. H. Kinney, arXiv:astro-ph/0301448.
[7] A. Lewis and S. Bridle, Phys. Rev. D 66, 103511 (2002) (available from http://cosmologist.info).

[8] Available at the LAMBDA Web site http://lambda.gsfc.nasa. gov/.

[9] C. L. Reichardt et al., arXiv:0801.1491.

[10] M. Cortes, A. R. Liddle, and P. Mukherjee, Phys. Rev. D 75, 083520 (2007).

[11] C. Destri, H. J. de Vega, and N. G. Sanchez, Phys. Rev. D 77, 043509 (2008).

[12] H. V. Peiris and R. Easther, arXiv:0805.2154. 